\title{
Complex neurological symptoms in bilateral thalamic stroke due to Percheron artery occlusion
}

This article was published in the following Dove Press journal:

Vascular Health and Risk Management

22 December 2016

Number of times this article has been viewed

\section{Paola Caruso \\ Paolo Manganotti \\ Rita Moretti}

Department of Clinical Neurology, University of Trieste, Trieste, Italy
Correspondence: Paola Caruso

Department of Clinical Neurology,

University of Trieste, Ospedale di

Cattinara, Strada di Fiume, 44734149

Trieste, Italy

Tel +39040 3994569

Fax +390404284

Email caruso.paola1983@libero.it

\begin{abstract}
The artery of Percheron is a rare anatomical variant where a single thalamic perforating artery arises from the proximal posterior cerebral artery (P1 segment) between the basilar artery and the posterior communicating artery and supplies the rostral mesencephalon and both paramedian territories of the thalami. Almost one-third of human brains present this variant. Occlusion of the artery of Percheron mostly results in a bilateral medial thalamic infarction, which usually manifests with altered consciousness (including coma), vertical gaze paresis, and cognitive disturbance. The presentation is similar to the "top of the basilar syndrome", and early recognition should be prompted. We describe the case of a young female with this vessel variant who experienced a bilateral thalamic stroke. Magnetic resonance angiography demonstrated bilateral thalamic infarcts and a truncated artery of Percheron. Occlusion of the vessel was presumably due to embolism from a patent foramen ovale. Thrombolysis was performed, with incomplete symptom remission, cognitive impairment, and persistence of speech disorders. Early recognition and treatment of posterior circulation strokes is mandatory, and further investigation for underlying stroke etiologies is needed.
\end{abstract}

Keywords: thalamus vascularization, cognitive impairment, paramedian thalamus territory, speech disorder, vertical gaze palsy

\section{Background}

The thalamus and midbrain have a complex blood supply with a large number of feeding arteries. The arterial supply of the thalamus and midbrain consists of a complex arterial network involving the anterior and posterior cerebral circulation. The anterior and inferior midbrain and thalami are supplied mostly by the internal carotid artery, while the medial, lateral, and posterior territories are irrorated from the vertebrobasilar system. In recent decades, according to pathological data, the following four territories for thalamic vascularization were identified: tuberothalamic, paramedian, inferolateral, and posterior choroidal arteries. The paramedian arteries normally supply the medial ventral thalami, hypothalamus, and subthalamic-mesencephalic junction and derive from the proximal segment of the posterior cerebral artery. ${ }^{1,2}$

In addition to the paramedian thalamic territory, the paramedian thalamic arteries supply the following medial areas of the upper brainstem: the interpeduncular nucleus, the decussation of the superior cerebellar peduncles, the medial part of the red nucleus, the third and fourth cranial nerve nuclei, and the anterior portion of the periaqueductal gray matter. ${ }^{3}$

Thalamic stroke accounts for $11 \%$ of vertebrobasilar infarcts. Strokes limited to paramedian territories account for $\sim 22-35 \%$ of all thalamic infarcts, usually due to 
cardioembolism. Paramedian arteries (arising from the P1 section of the posterior cerebral artery on both sides) supply dorsomedian nucleus (divided into anteromedial magnocellular and posterolateral parvocellular mediodorsal nuclei that receive projections from the amygdala and are connected to the prefrontal cortex and ventral pallidum) and the intralaminar nuclei (central lateral, centromedian, and parafascicular; the intralaminar nuclei are divided into the caudal part and the rostral part that project to the orbitofrontal and mediofrontal cortices, the motor and premotor cortex, and the internal globus pallidus). ${ }^{4-6}$

Consequently, occlusion of the artery of Percheron causes a bilateral paramedian thalamic infarction with or without midbrain infarction. Additional involvement of the anterior thalamus is uncommon.

Regarding the origin of the paramedian artery, it is known that in one-third of human brains, the vessel originates from a single pedicle named the type B artery of Percheron. In these cases, stroke affects both paramedian thalamic regions and may lead to a complex clinical syndrome $(\sim 0.1-2 \%$ of all ischemic strokes; Figure 1). ${ }^{7}$

The artery of Percheron may be visualized with CT or MR angiography. However, diagnosis of Percheron artery infarction is often made in the late stage. Successful recanalization of the artery of Percheron with thrombolysis has been described.

In the majority of cases, clinical features include varying levels of decreased consciousness, neuropsychological and memory impairment, and vertical gaze palsy. Oculomotor disturbances, hemiplegia, cerebellar ataxia, and movement disorders have also been described. ${ }^{8}$

The goal of this article is to report a case where the diagnosis of an infarction due to an occlusion of the Percheron artery has been made directly and not retrospectively as usually appears.

\section{Case presentation}

We describe the case of a 52-year-old well-fit Caucasian female who was admitted to our emergency department because of a sudden onset of decreased consciousness and speech disorder. Written informed consent was received from the patient for this study. The first neurological examination revealed a Glasgow Coma Scale score of 8 and a National Institutes of Health Scale Score of 13.

When deeply stimulated, she could only open her eyes, verbal communication was absent, and she was not able to perform any command, and on cranial nerve examination, vertical gaze palsy was detected, while no other motor deficits could be identified. Blood examinations excluded a possible metabolic, toxic, infective, endocrine, or iatrogenic etiology of the comatose state.

Brain CT scan examination was immediately obtained, and no acute lesions could be seen; a filling defect of the P1 segment of the left posterior cerebral artery was only described, and the radiologist could not identified the posterior communicant left artery (Figure 2).

However, taking into account all the symptoms and considering the possibility of an acute stroke, intravenous thrombolysis was performed resulting in a slow improvement of her clinical condition in the next days, although the confusion state, disorientation, slurred speech, and oculomotor disorder persisted.

On the next day, thrombolysis brain CT and MRI scans were performed that showed bilateral thalamic lesions in the paramedian territory (Figure 3).

During recovery, duplex ultrasound of trial sequential analysis and transcranial doppler ultrasound and transesophageal echocardiography were performed that revealed a patent foramen ovale with spontaneous passage of contrast bubbles from the right auricle to the left cavities, which could probably be the cause of embolism.

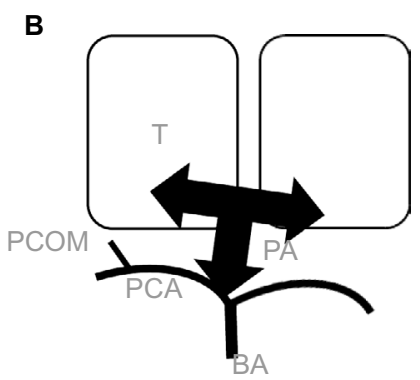

Figure I (A) Schematic representation of the affected thalamic nuclei in this case compared to an axial diffusion-weighted magnetic resonance image of the same patient. The black line limits the area of infarction in both paramedian regions. (B) Schematic representation of the artery of PA, T, BA, PCA, and PCOM. Abbreviations: PCOM, posterior communicating artery; BA, basilar artery; CM, centromedian; CL, central lateral; MD, dorsomedialis; Pf, parafascicularis; PA, percheron; PCA, posterior cerebral artery; $\mathrm{Pv}$, paraventricular; $\mathrm{T}$, thalamus. 


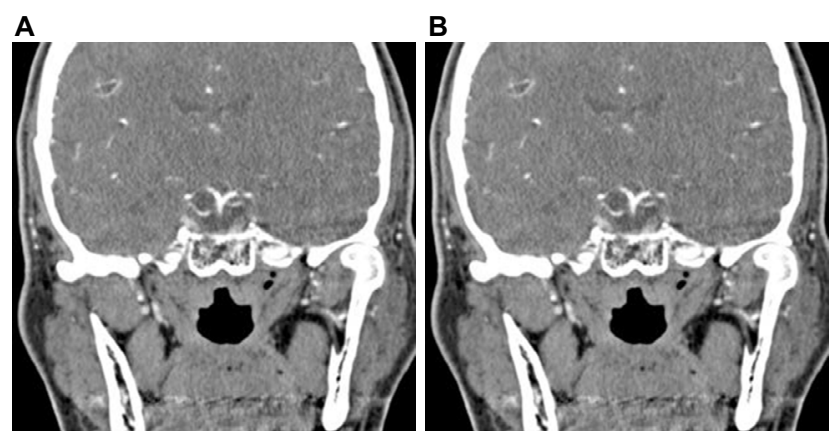

Figure 2 Brain angio CT scan's coronal view $(\mathbf{A})$ and axial view (B). Note: Performed in the emergency department, showing no parenchymal lesions or main vessel obstruction: brain CT scan examination showed only a filling defect of the $\mathrm{PI}$ segment of the left posterior cerebral artery $(\mathbf{A})$.
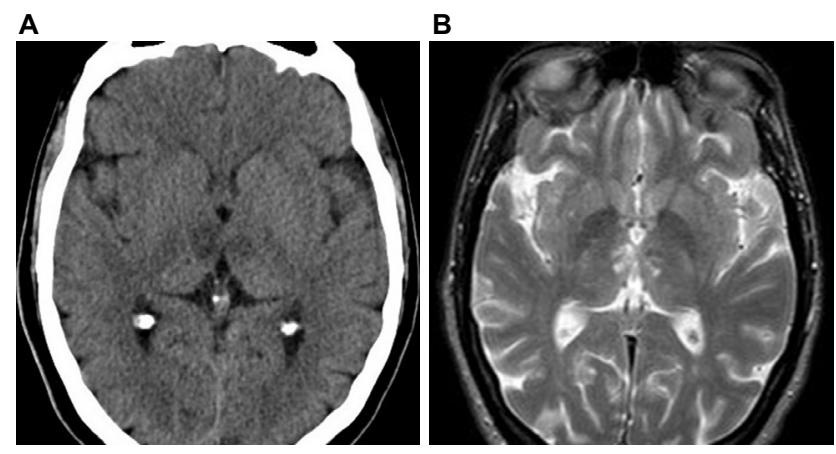

Figure 3 (A) Brain CT scan axial view, performed after thrombolysis showing ischemic stroke in the paramedian territory of both thalami. (B) MRI axial view seq $\mathrm{T} 2$.

The first neuropsychological exam showed deficits on attention and memory task changes in behavioral were found, like impulsive behavior, also deficit in executive function were found, anosoagnosia and slowness in executive functions.

After a week, the patient was discharged to home with a diagnosis of a bilateral thalamic stroke in cardioembolism. After 3 months, she was evaluated again and memory and behavioral impairment could be still detected. She was not able to go back to her work.

\section{Discussion}

First described in 1973, the artery of Percheron is a rare anatomical variant where a single thalamic perforating artery arises from the proximal posterior cerebral artery (P1 segment) between the basilar artery (BA) and the posterior communicating artery and supplies the rostral mesencephalon and both paramedian thalami. If this artery becomes occluded, bilateral paramedian thalamic infarction occurs. Proximal embolism is thought to be the most common etiology of stroke in this territory with this variant. Asymmetric thalamic involvement is seen in two-thirds of cases, and midbrain infarction is present in over half. ${ }^{9}$
Conventional vascular imaging does not routinely demonstrate these tiny perforating vessels. Hypoplastic or absent P1 segments are more likely to be seen with this variant.

Lazzaro et al identified four patterns of ischemic infarctions when the Percheron artery is occluded. Approximately $43 \%$ of their cases presented infarction of both paramedian thalami and midbrain, and in $38 \%$ of cases, only damage to paramedian thalami was detected. In $\sim 14 \%$ of patients, the damage involved the anterior thalamic nuclei in addition to paramedian thalami and upper midbrain. The least common pattern (5\%) was ischemic damage of the bilateral paramedian and anterior thalami; the midbrain was spared in these cases. Moreover, in their work, the "V" sign for the diagnosis of Percheron artery was described; a fluid-attenuated inversion recovery (FLAIR) and diffusion weight imaging sequences in $67 \%$ of cases of artery of Percheron infarction with midbrain involvement were found, and this sign supports the diagnosis when present. The "V" sign appears as a distinct pattern of V-shaped hyperintensity on axial FLAIR and/or diffusion weight imaging along with the pial surface of the midbrain adjacent to the interpeduncular fossa. ${ }^{10}$

Although bilateral ischemic lesions can be seen on MRI, differential diagnoses include inflammatory, infectious, and neoplastic illnesses; particularly, venous infarction and neoplastic lesion do not follow a particular arterial territory.

However, more often, diagnosis is only clinical; actually, patients with bilateral paramedian thalamic lesions may develop altered sensorium, vertical gaze palsy, and memory impairment. Sudden onset of altered eye movements with partial/complete limitation of vertical gaze, loss of convergence, bilateral internuclear ophthalmoplegia, pseudopalsy of the abducens nerve, and intolerance to light are the characteristics; pupils are frequently abnormal, and pupil size varies depending on the involvement of the Edinger-Westphal nucleus or oculomotor fascicles, descending sympathetic fibers, or some combination of these structures. ${ }^{11-13}$ Dysarthria, altered consciousness, and motor paresis should alert the clinician to the possibility of ischemia of posterior circulation territory, including the BA. Severe cognitive impairment, amnesic syndrome, and executive dysfunction impairment can occur, which can still be present some months after presentation. Behavioral problems with inappropriate social conduct, apathy, and flattened emotions are also described. ${ }^{14,15}$

Recently, Arauz et al, ${ }^{16}$ in a study of 15 consecutive patients with a Percheron artery infarct, described the following seven main clinical patterns: 1) mental status disturbance; 2) behavioral amnesic impairment (confusion, agitation, apathy, or disinhibition); 3) aphasia or dysarthria including any component of language component or slurred speech; 4) ocular movements; 5) motor deficits; 6) cerebellar signs; 
and 7) other symptoms that are not previously reported (such as hypersomnia, tremors, seizures, and hyperthermia).

The large number of variants of the blood supply in the posterior cranial fossa (especially regarding the presence and size of P1 segments, which give rise to the paramedian arteries) might show that the artery of Percheron is not such an infrequent variant and is most underdiagnosed. The characteristic artery of Percheron infarction may occur in $0.1-2 \%$ of all ischemic strokes and in 4-18\% of all thalamic strokes.

Stroke due to PA artery occlusion should be suspected in every patient with acute onset of symptoms related to posterior circulation deficit. Diagnosis is firstly clinical, acute neuroimaging may not help in diagnosis, main clinical signs include vertical gaze palsy, alteration in consciousness and cognition. If possible intravenous thrombolysis should be performed, although the intraarterial approach has been described with successful exit.

\section{Disclosure}

The authors report no conflicts of interest in this work.

\section{References}

1. Carrera E, Michel P, Bogousslavsky J. Anteromedian, central and posterolateral territory infarcts: three variant types. Stroke. 2004; 35(12):2826-2834.

2. Schmahmann J. Vascular syndromes of the thalamus. Stroke. 2003; 34(9):2264-2278.

3. Lamot U, Ribaric I, Popovic KS. Artery of Percheron infarction: review of literature with a case report. Radiol Oncol. 2015;49(2):141-146.
4. Castaigne P, Lhermitte F, Buge A, Escourolle R, Hauw JJ, Lyon-Caen O. Paramedian thalamic and midbrain infarcts: clinical and neuropathological study. Ann Neurol. 1981;10(2):127-148.

5. Bogousslavsky J, Van Melle G, Regli F. The Lausanne Stroke Registry: analysis of 1,000 consecutive patients with first stroke. Stroke. 1988;19(9):1083-1092.

6. Bogousslavsky J, Regli F, Uske A. Thalamic infarcts: clinical syndromes, etiology, and prognosis. Neurology. 1988;38(6):837-847.

7. Saez de Ocariz M, Nader J, Santos J, Bautista M. Thalamic vascular lesions. Risk factors and clinical course for infarcts and hemorrhages. Stroke. 1996;27(9):1530-1536.

8. Cassourret G, Prunet B, Sbardella F, Bordes J, Maurin O, Boret H. Ishemic stroke of the artery of Percheron with normal initial MRI: a case report. Case Rep Med. 2010;2010:425734.

9. Percheron G. Arteries of the human thalamus: II. Arteries and paramedian thalamic territory of the communicating basilar artery. Rev Neurol (Paris). 1976;132(5):309-324.

10. Lazzaro NA, Wright B, Castillo M, et al. Artery of Percheron infarction: imaging patterns and clinical spectrum. AJNR Am J Neuroradiol. 2010;31(7):1283-1289.

11. Thurtell MJ, Halmagyi GM. Complete ophthalmoplegia an unusual sign of bilateral paramedian midbrain-thalamic infarction. Stroke. 2008;39(4):1355-1357.

12. Amin OSM, Shwani SS, Zangana HM, Hussein EMH, Ameen NA. Bilateral infarction of paramedian thalami: a report of two cases of artery of Percheron occlusion and review of the literature. BMJ Case Rep. 2011;2011.

13. Turner J, Richardson T, Kane I, Vundavalli S. Decreased consciousness: bilateral thalamic infarction and its relation to the artery of Percheron. BMJ Case Rep. 2014;2014.

14. Jiménez Caballero PE. Bilateral paramedian thalamic artery infarcts: report of 10 cases. J Stroke Cerebrovasc Dis. 2010;19(4):283-289.

15. Margolin E, Hanifan D, Berger MK, Ahmad OR, Trobe JD, Gebarski SS. Skew deviation as the initial manifestation of left paramedian thalamic infarction. J Neuroophthalmol. 2008;28(4):283-286.

16. Arauz A, Paino-Rodriguez HM, Vargas-Gonzalez JC, et al. Clinical spectrum of artery of Percheron infarct: clinical-radiological correlations. J Stroke Cerebrovasc Dis. 2014;23(5):1083-1088.
Vascular Health and Risk Management

\section{Publish your work in this journal}

Vascular Health and Risk Management is an international, peerreviewed journal of therapeutics and risk management, focusing on concise rapid reporting of clinical studies on the processes involved in the maintenance of vascular health; the monitoring, prevention and treatment of vascular disease and its sequelae; and the involvement of

\section{Dovepress}

metabolic disorders, particularly diabetes. This journal is indexed on PubMed Central and MedLine. The manuscript management system is completely online and includes a very quick and fair peer-review system, which is all easy to use. Visit http://www.dovepress.com/ testimonials.php to read real quotes from published authors. 